\title{
Contactless Ultrasonic Treatment in Direct Chill Casting
}

\author{
CATHERINE E. H. TONRY ${ }^{1,2},{ }^{1,2}$ VALDIS BOJAREVICS ${ }^{1},{ }^{1}$ \\ GEORGI DJAMBAZOV $10,{ }^{1}$ and KOULIS PERICLEOUS ${ }^{1}{ }^{1}$ \\ 1.-Computational Science and Engineering Group, University of Greenwich, Park Row, London \\ SE10 9LS, UK. 2.-e-mail: c.tonry@greenwich.ac.uk
}

\begin{abstract}
Uniformity of composition and grain refinement are desirable traits in the direct chill (DC) casting of non-ferrous alloy ingots. Ultrasonic treatment is a proven method for achieving grain refinement, with uniformity of composition achieved by additional melt stirring. The immersed sonotrode technique has been employed for this purpose to treat alloys both within the launder prior to DC casting and directly in the sump. In both cases, mixing is weak, relying on buoyancy-driven flow or in the latter case on acoustic streaming. In this work, we consider an alternative electromagnetic technique used directly in the caster, inducing ultrasonic vibrations coupled to strong melt stirring. This 'contactless sonotrode' technique relies on a kilohertz-frequency induction coil lowered towards the melt, with the frequency tuned to reach acoustic resonance within the melt pool. The technique developed with a combination of numerical models and physical experiments has been successfully used in batch to refine the microstructure and to degas aluminum in a crucible. In this work, we extend the numerical model, coupling electromagnetics, fluid flow, gas cavitation, heat transfer, and solidification to examine the feasibility of use in the DC process. Simulations show that a consistent resonant mode is obtainable within a vigorously mixed melt pool, with high-pressure regions at the Blake threshold required for cavitation localized to the liquidus temperature. It is assumed that extreme conditions in the mushy zone due to cavitation would promote dendrite fragmentation and coupled with strong stirring, would lead to fine equiaxed grains.
\end{abstract}

\section{INTRODUCTION}

Ultrasonic treatment (UST) of melts has been shown to lead to degassing and grain refinement. ${ }^{1-3}$ Grain refinement is necessary in direct chill (DC) casting to prevent hot tearing, ${ }^{4}$ shrinkage porosity, ${ }^{5}$ and cold cracking. ${ }^{6}$ A fine grain structure gives improved material properties to the metals, and also facilitates subsequent mechanical working. Columnar to equiaxed transition $(\mathrm{CET})^{7,8}$ is required to achieve a uniform fine grain structure in the core of the ingot. There are many methods to achieve CET, with the most common practice relying on grain refiners. ${ }^{9}$ Alternative methods used to promote CET include melt conditioning, ${ }^{10}$ electric current pulse ${ }^{11}$ pulse magneto-oscillation, ${ }^{12}$ pulse magnetic field, ${ }^{13}$ electromagnetic (EM) stirring, ${ }^{14}$ and, as discussed here, UST.
There have been many investigations of UST of alloys in DC casting, ${ }^{1,15-18}$ where the focus is typically on treating the melt prior to casting, either in a crucible ${ }^{15}$ or in the launder. ${ }^{17}$ Recent investigations have included a sonotrode inside the sump ${ }^{18}$ treating the melt as it is solidifying. In Lebon et al., ${ }^{18}$ main observations were a change in flow pattern due to acoustic streaming leading to a higher temperature gradient in the transition zone, reducing its size. Although crystallization was not modeled, parallel experiments demonstrated grain refinement in the cast billet. The primary disadvantage of an immersed sonotrode is that limited volumes can be treated, and consequently multiple sonotrodes were required for large castings, as shown by Eskin and Eskin. ${ }^{1}$ The use of an electromagnetic coil as a contactless source of ultrasound has been demonstrated to work in aluminum crucibles ${ }^{19,20}$ containing up to $10.5 \mathrm{~kg}$ of metal. This process, using a 
high-frequency induction coil suspended close to the liquid-free surface ${ }^{21}$ resulted in grain refinement and degassing after 2-4 min of treatment. Given that the high-frequency field does not penetrate the melt for more than a few millimeters, acoustic resonance is sought to achieve the pressure threshold required for cavitation in the treatment volume. The time-averaged component of the Lorentz force introduces the added benefit of electromagnetic stirring to aid mixing and prevent macro-segregation. Since the coil does not contact the metal surface, the risk of contamination and probe erosion faced by the traditional immersed sonotrode is avoided and, unlike immersed sonotrode techniques, the active cavitation zone was shown to lie deep in the melt pool rather than in the immediate area surrounding the probe, reducing the effects of gas shielding. Finally, large volumes can be treated effectively by designing a suitable induction coil and ensuring that acoustic resonance is reached by tuning the current frequency. This paper focuses on numerical modeling efforts to test the feasibility of using the top-coil in vertical DC casting, taking into consideration the geometry and the continuous nature of this process.

Recent work by Kaldre et al. ${ }^{22}$ used a high-frequency coil combined with a permanent magnetic field to achieve a similar effect. The use of electromagnetic fields in DC casting is, of course, not new, but their function has been quite different, used, for example, at a lower frequency for stirring to control segregation, such as the work by Hatic et al. ${ }^{23}$

\section{METHODOLOGY}

Three separate codes are used in this work. The first 'SPHINX', is an in-house spectral collocation code, used to calculate the coupling between solidification, temperature, magnetohydrodynamic flow ${ }^{24}$ and surface deformation due to the EM force. The other two models provide alternative solutions of the sound field. The second uses the finite element analysis package COMSOL Multiphysics 5.2 to predict acoustic resonant modes in the frequency domain, and the third, part of the in-house finite volume suite Physica, predicts transient acoustic cavitation and the instantaneous velocity field produced by soundwaves. Key information, such as the temperature to calculate the speed of sound and resonant modes to impose the driving frequency, are passed between models. It is assumed there is no feedback between the acoustic flow field and the mean flow, so the information transport between SPHINX and Physica is one way only. Figure 1 demonstrates the relationships between them. The geometry used represents the experimental DC caster installed at BCAST ${ }^{18}$ shown in Fig. 2. In both acoustic models, the speed of sound is assumed to have a linear relationship with the solid fraction in the mushy zone, and to be the same as that of pure aluminum. The liquid fraction and densities were calculated for AA6060 using Thermo-Calc as seen in Fig. 3. Material properties used in the models can be seen in Table I, and a full description for each model is given in the following subsections.

\section{Fluid Flow and Temperature Model}

The time-dependent conservation equations for mass, momentum, and energy are solved, accounting for the effects of electromagnetic interaction and change of phase. An in-house software SPHINX solves the equations numerically using a spectral colocation technique ${ }^{25}$ on a continuously deformable mesh coincident with the metal volume, as described in Ref. 24. The method allows real-time deformation of the liquid-free surface based on the instantaneous balance of the forces acting. The turbulent time dependent flow is governed by the momentum conservation and the incompressible fluid continuity equations:

$$
\partial_{t} \mathbf{v}+(\mathbf{v} \cdot \nabla) \mathbf{v}=-\rho^{-1} \nabla p+\nabla \cdot\left(v_{e}\left(\nabla \mathbf{v}+\nabla \mathbf{v}^{T}\right)\right)+\rho^{-1} \mathbf{f}_{m}+S_{D},
$$

$$
\nabla \cdot \mathbf{v}=0
$$

where $\mathbf{v}$ is the velocity, $\rho$ the density, $p$ the pressure, $v_{e}$ the effective viscosity (sum of laminar, $v$, and turbulent viscosity, $v_{T}$, contributions), $\mathbf{f}_{\mathrm{m}}$ the volumetric electromagnetic force, and $S_{D}$ a Darcy flow resistance term that accounts for the macroscopic effect of phase change on flow in mushy zones. Buoyancy has been ignored in this problem since its effect is small compared to the electromagnetic force.

The boundary conditions assume no-slip at solid walls $(v=0)$, and free surface dynamic and kinematic conditions when the liquid metal is detached from any solid surface. ${ }^{26}$

The temperature variation with the EM coil moving towards the melt is described by the energy conservation equation:

$$
\frac{\partial\left(\rho C_{p} T\right)}{\partial t}+\nabla \cdot\left(\rho C_{p} \mathbf{v} T\right)=\nabla \cdot\left(k_{e f f} \nabla T\right)+q_{e}+q_{L}
$$

where $T$ is the temperature, $C_{p}$ the specific heat, and $k_{\text {eff }}$ the effective thermal conductivity accounting for the turbulence in the flow. The last two terms in Eq. 3 represent Joule heating, $q_{e}$, and latent heat release, $q_{L}$, respectively. As given in Eq. 4:

$$
q_{e}=\frac{\mathbf{J}^{2}}{\sigma}, q_{L}=-\frac{\partial}{\partial t}\left(\rho f_{L} L\right)-\nabla \cdot\left(\rho \mathbf{v} f_{L} L\right)
$$

where $\mathbf{J}$ is the electric current density induced in the liquid, $\sigma$ the electrical conductivity of the metal, and $L$ the solidification latent heat. The coupled electromagnetic field is computed according to the dynamic mutual induction algorithm described in previous publications. ${ }^{27}$ 
Table I. Material properties used in simulation

\begin{tabular}{|c|c|c|c|c|c|}
\hline Material & $\begin{array}{c}\text { Electrical } \\
\text { conductivity (S/m) } \\
\end{array}$ & $\begin{array}{r}\text { Density } \\
\left(\mathbf{k g} / \mathbf{m}^{3}\right) \\
\end{array}$ & $\begin{array}{c}\text { Speed of sound } \\
(\mathbf{m} / \mathbf{s})\end{array}$ & $\begin{array}{c}\text { Surface tension } \\
(\mathbf{N} / \mathbf{m}) \\
\end{array}$ & $\begin{array}{c}\text { Dynamic viscosity } \\
(\mathbf{P a} \cdot \mathbf{S})\end{array}$ \\
\hline Aluminum melt & $2 \times 10^{6}$ & 2375 & 4560 & 0.87 & 0.0013 \\
\hline Aluminum solid & & & 6400 & & \\
\hline Air & $1 \times 10^{-8}$ & 1.2 & 343 & & \\
\hline
\end{tabular}

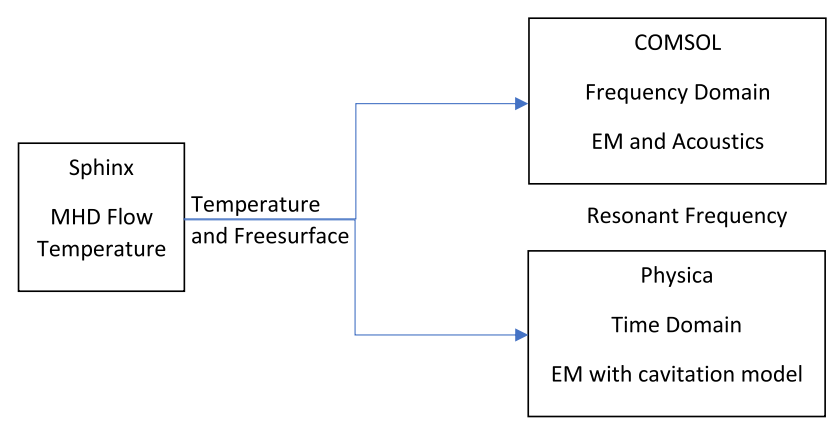

Fig. 1. Diagram of the loose coupling between codes.

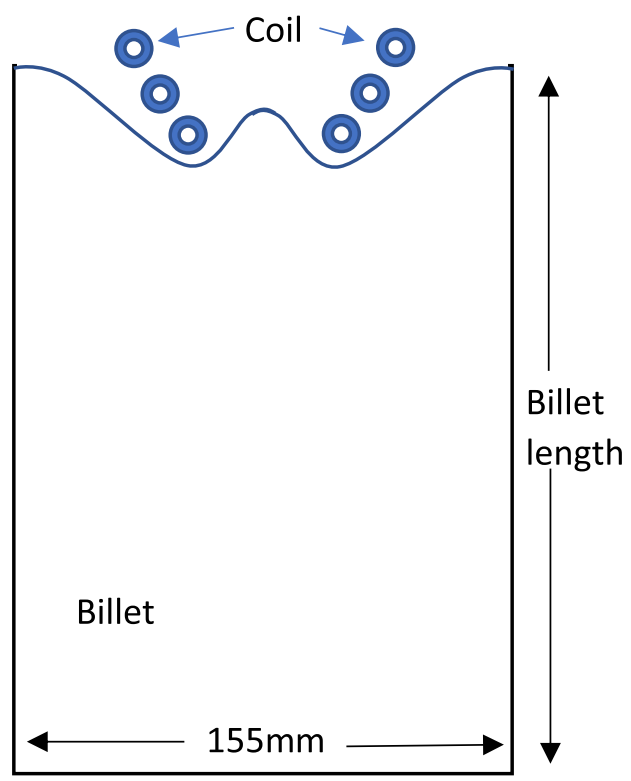

Fig. 2. Schematic of the geometry.

The liquid fraction, $f_{S}$, is approximated from the data shown in Fig. 3 as a function of temperature $T$ :

$f_{S}=\left\{\begin{array}{lll}0 & T>T_{L} & \text { liquid } \\ A_{L}+B_{L} T+C_{L} T^{2}+D_{L} T^{3} & T_{S} \leq T \leq T_{L} & \text { mushyzone } \\ 1 & T<T_{S} & \text { solid }\end{array}\right.$

the coefficients $A_{L}, B_{L}, C_{L}$, and $D_{L}$ are also given in Fig. 3 Then, the Darcy flow resistance term in Eq. 1 is specified as:

$$
S_{D}=\frac{\mu}{K} v, K=\frac{f_{L}^{3}}{\zeta\left(1-f_{L}\right)^{2}},
$$

where $f_{L}=1-f_{S}$. The heat transfer boundary conditions are stated for free surface radiation and wall loss, described in Ref 24. The effective heat transfer at solid walls is then given by:

$$
-\rho C_{p} \alpha_{e} \partial_{n} T=h\left(T-T_{w}\right)
$$

where $h(T)$ is the heat transfer coefficient ( $\mathrm{see}^{26}$ for experimental validation of the specific values used).

In the spray-cooling zone:

$$
\begin{aligned}
& h=50, T<T_{S}, \\
& h=50+200\left(T-T_{S}\right), T \geq T_{S},
\end{aligned}
$$

where $T_{S}$ is the metal solidus temperature, and, above this, $h=10\left(\mathrm{Wm}^{-2} \mathrm{~K}^{-1}\right)$.

At the free surface, radiation loss is given by:

$$
-\rho C_{p} \alpha_{e} \partial_{n} T=\varepsilon \sigma_{R}\left(T^{4}-T_{w}^{4}\right)
$$

The distribution of effective turbulent thermal diffusivity, $\alpha_{e}$, is computed using the $k-\omega$ turbulence model of Wilcox ${ }^{28}$ :

$$
\left.\begin{array}{l}
\partial_{t} k+\mathbf{v} \cdot \nabla k=\nabla \cdot\left[\left(v+\sigma_{k} v_{T}\right) \nabla k\right]+G-\beta^{*} \omega k \\
\partial_{t} \omega+\mathbf{v} \cdot \nabla \omega=\nabla \cdot\left[\left(v+\sigma_{\omega} v_{T}\right) \nabla \omega\right]+\alpha \frac{\omega}{k} G-\beta \omega^{2} \\
v_{T}=\alpha^{*} \frac{k}{\omega}
\end{array}\right\}
$$

where $\omega$ is the frequency of vorticity fluctuations, $k$ the turbulence kinetic energy per unit mass, and the various constants are functions of $R_{T}$, the local turbulent Reynolds number (see Wilcox ${ }^{28}$ ).

\section{Acoustic Resonance Model}

The resonant modes are predicted using a oneway coupled electromagnetic acoustic model. This model, developed in COMSOL Multiphysics 5.2, has been validated for use in crucibles. ${ }^{19}$ The model solves both the electromagnetic field and the acoustic response in the frequency domain. To solve the electromagnetic field, a 2D axisymmetric model is used for solving the full Maxwell equations with a current in the coil approximated by: 

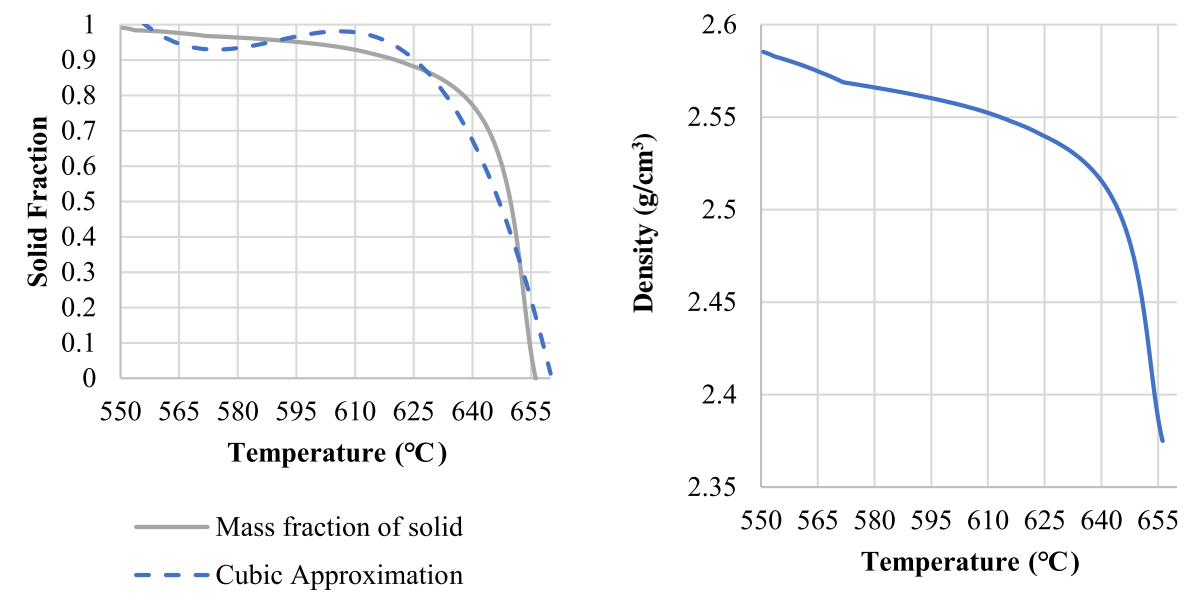

\begin{tabular}{|c|c|c|c|}
\hline$A_{L}$ & $B_{L}$ & $C_{L}$ & $D_{L}$ \\
\hline $6.7343463590230522 \mathrm{e} 2$ & -3.4229653849793067 & $5.8035442115111730 \mathrm{e}-3$ & $-3.2775707576431694 \mathrm{e}-6$ \\
\hline
\end{tabular}

Fig. 3. The temperature-dependence of the solid fraction and density for A6060.

$$
I_{\text {coil }}=\mathbf{J} \cdot \mathbf{e}_{\text {coil }} \mathrm{d} r \mathrm{~d} z, \mathbf{J}_{\mathbf{e}}=\sigma \frac{V_{\text {coil }}}{2 \pi r} \mathbf{e}_{\text {coil }},
$$

where $I_{\text {coil }}$ is the out-of-plane coil current, $\boldsymbol{e}_{\text {coil }}$ an out-of-plane unit vector, $J$ the current density in the coil, $\mathbf{J}_{\mathbf{e}}$ the external current density and $V_{\text {coil }}$ the voltage in the coil. The electromagnetic field is solved in the coil, the melt, and the surrounding material (assumed to be air), with far field boundary conditions set to magnetic and electric insulation. The magnetic flux density obtained from this model is then used to calculate the magnetic pressure ${ }^{19}$ :

$$
p_{b}=\frac{\mathbf{B}^{2}}{2 \mu_{0}}
$$

where $p_{b}$ is the background pressure, $\mathbf{B}$ the magnetic flux density, and $\mu_{0}$ the permeability of free space. The pressure acoustics are then solved in the frequency domain using the Helmholtz equation:

$$
\begin{gathered}
\nabla \cdot\left(-\frac{1}{\rho_{c}}\left(\nabla p_{t}\right)\right)-\frac{k_{\mathrm{eq}}^{2} p_{t}}{\rho}=0, \\
p_{t}=p+p_{b}, \\
k_{\mathrm{eq}}^{2}=\left(\frac{\omega}{c}\right)^{2}
\end{gathered}
$$

where $\rho$ is the density, $p$ the acoustic pressure, $p_{\mathrm{t}}$ the total acoustic pressure, $\omega$ the angular frequency, and $c$ the effective speed of sound in the medium. The acoustic field is only solved in the melt, with sound soft boundaries at the melt surface and the edges of the billet.

\section{Time-Dependent Acoustic Cavitation Model}

Based on the direct numerical simulation of sound waves, the time-dependent acoustic cavitation model meets the objective of predicting the influence of cavitation on the ultrasound field and, in particular, the effect cavitation has on resonance in the melt. That influence occurs during the rarefaction part of the acoustic cycle, when the bubbles expand to hundreds of times their original size, while during compression, the volume occupied by the bubbles is negligible and the elasticity of the melt is determined by the compressibility of the liquid itself. For this reason (and to avoid computationally intensive resolution of very high frequencies), the shock waves resulting from bubble collapses are not included in this model, but their overall effect can be taken into account by adding dissipation into the equations.

Inertial cavitation occurs in high-intensity ultrasound processing where the vacuum during the negative phase of the acoustic cycle is sufficiently deep to overcome the surface tension on the interface between an existing hydrogen bubble and the liquid metal, and to cause rapid, explosive expansion of the bubble. Such acoustic amplitudes are referred to as being above the Blake threshold. ${ }^{29}$ The growing (and subsequently contracting) voids act locally as attenuating sources of sound, because they relax the tension in the liquid during the rarefaction half-cycle, diminishing the overall acoustic amplitude.

Mathematically, this phenomenon can be modeled by introducing the concept of the time-dependent bubble volume fraction, $\beta=4 / 3 \pi R^{3} n$, which depends on the bubble radius, $R$, and the concentration of bubbles, $n$, both considered as field variables in a continuum model based on acoustic approximations of the fluid mechanics equations ${ }^{30}$ : 
Mass conservation

$$
\frac{1}{\rho_{L} c_{L}^{2}} \frac{\partial p}{\partial t}+\nabla \cdot \mathbf{u}-\frac{\partial \beta}{\partial T}=0
$$

Momentum equation

$$
\rho_{L} \frac{\partial \mathbf{u}}{\partial t}+\nabla p=0
$$

Gas state equation

$$
p_{g}=\kappa\left(\frac{M}{\frac{4}{3} \pi R^{3}}\right)^{\gamma}
$$

Rayleigh-Plesset equation

$$
R \ddot{R}+\frac{3}{2} \dot{R}^{2}=\frac{1}{\rho_{L}}\left(p_{g}-p\right) .
$$

The bubbles in the above Caflisch equations can contain vapor or gas (e.g., hydrogen coming out of solution during ultrasound treatment of liquid aluminum) or both (e.g., in water model experiments aimed at visualizing the process). The state of that gas mixture can, for most practical cases with inertial cavitation, be modeled with some form of an effective ideal gas-law equation, as shown above with constants, $\kappa, M$, and $\gamma$, for the initial gas state, mass of gas in the bubble, and polytropic index, respectively. The oscillations of the bubble radius, driven by the time-dependent absolute local pressure, $p$, and influenced by the inertia of the surrounding liquid, can be described by the RayleighPlesset equation (Eq. 19) with over-dots representing ordinary temporal derivatives. Improved bubble dynamics models, e.g., Keller-Miksis equations, ${ }^{31}$ result in accurate representation of the after-bounces following a bubble collapse, but show little or no difference over the period of rapid bubble growth and contraction until the moment of the main bubble collapse.

A new, practical approach for solving the tightlycoupled bubble dynamics and acoustic equations in the Caflisch model is proposed here. The aim is to adequately represent the influence of inertial cavitation on the sound field in the frequency range of interest, which includes the driving ultrasound frequency and several of its lowest harmonics, but not the very high-frequency or broadband noise produced by stably vibrating bubbles.

The capability of the model to predict the ultrasound intensity under the conditions of strong, inertial cavitation is especially important in cases where resonance amplification of the acoustic amplitude is desired, as in an electromagnetic, contactless generation of ultrasound for liquid metal processing.

Where estimation of the high-frequency or broadband noise is also required, additional software modules based on the linear propagation of acoustic waves emitted from the collapsing and rebounding bubbles can be attached to the implementation described here. The theoretical expressions for sound waves from point sources in a

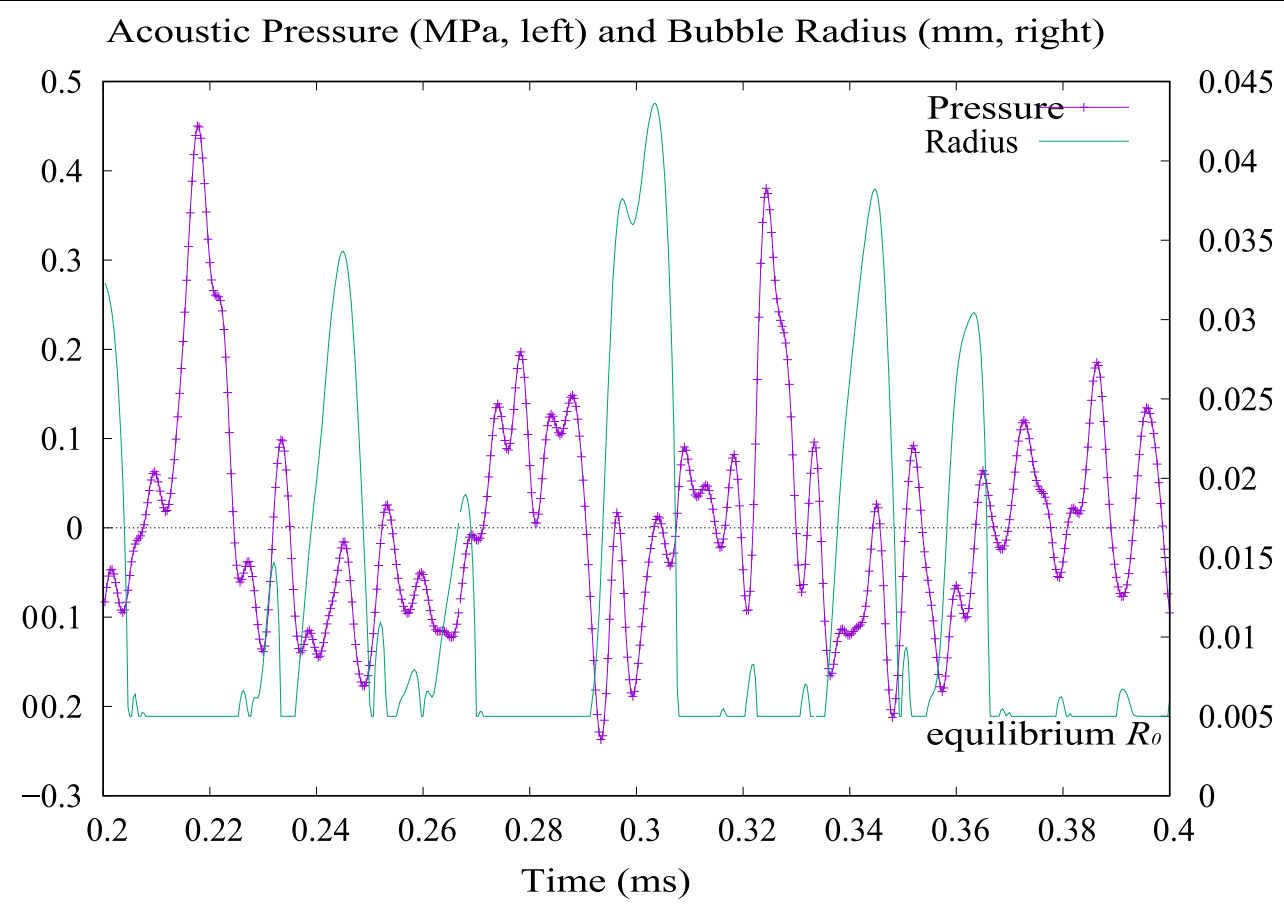

Fig. 4. Cavitation model behavior for an acrylic tank. 

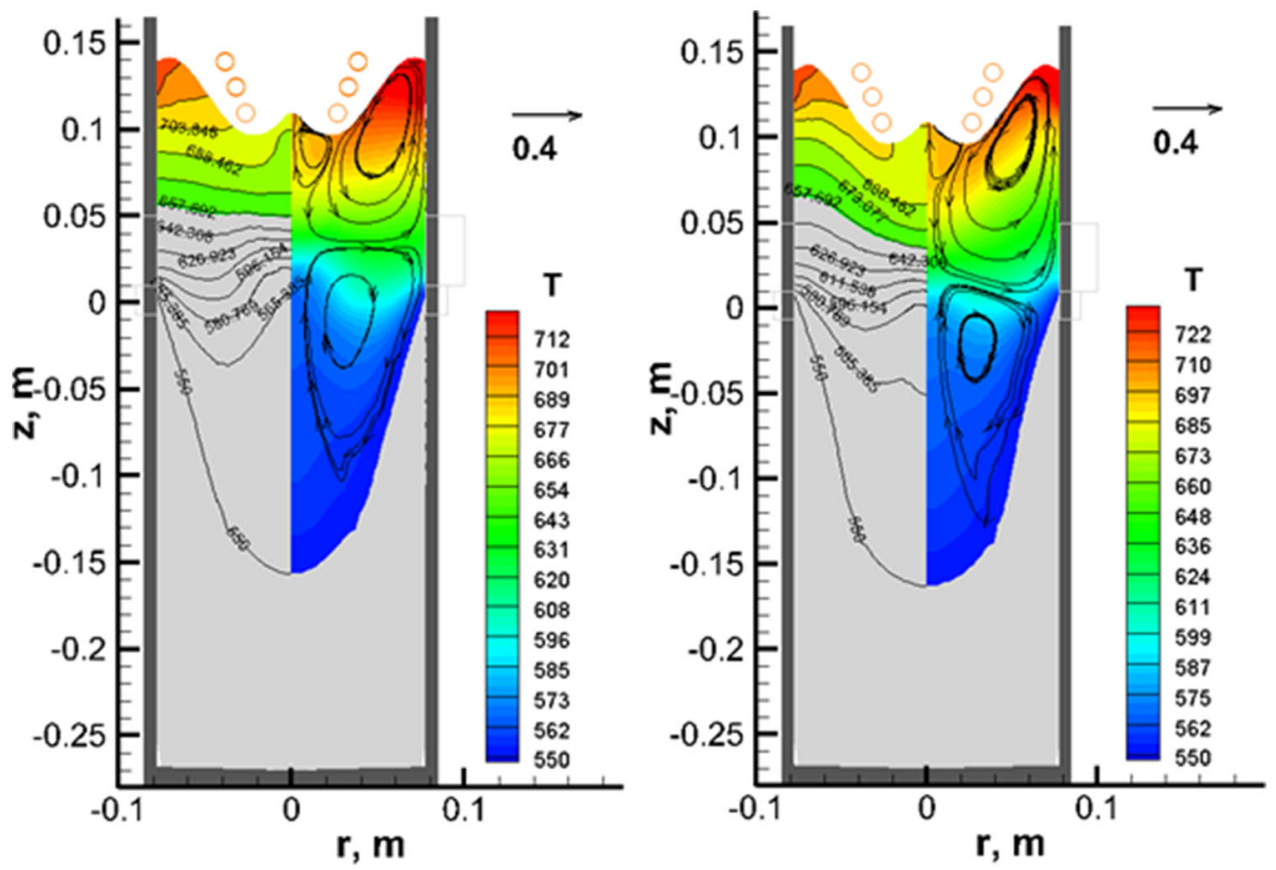

Fig. 5. Temperature (solidus and liquidus line cut-outs) and flow fields obtained using SPHINX: coil current $2000 \mathrm{~A}$ at AC frequency 8800 (left) and $15,000 \mathrm{~Hz}$ (right).

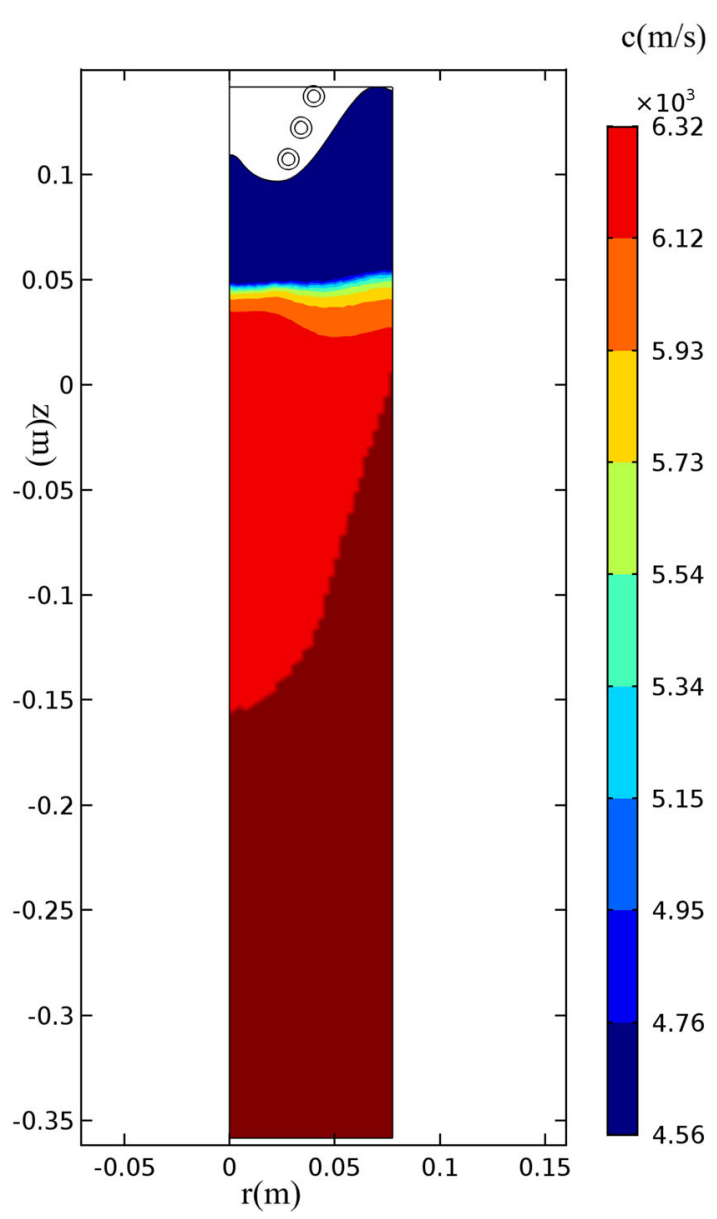

Fig. 6. Demonstration of the change in the speed of sound in the melt pool.

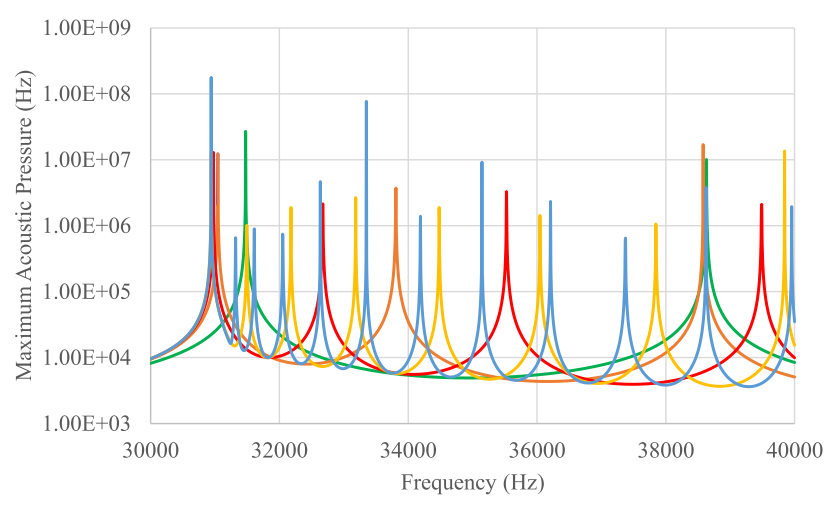

$-0.25 \mathrm{~m}$ Billet $-0.39 \mathrm{~m}$ Billet $-0.5 \mathrm{~m}$ Billet $-1 \mathrm{~m}$ Billet $-1.5 \mathrm{~m}$ Billet

Fig. 7. The frequency response of the frequency domain model.

uniform medium can be used with these 'additional' high-frequency waves, and the results superimposed on the main acoustic field which is strongly influenced by the cavitation.

The proposed coupled acoustic-bubbles model for the main acoustic field comprises the following stages: (1) solve the acoustic partial differential equations (PDEs) (mass continuity and momentum) time-dependently, with a time step appropriate for resolving the highest frequency in the desired range; (2) at each acoustic step, make an ordinary differential equation (ODE) step with the RayleighPlesset equation for all representative bubbles located at the centers of the acoustic computational cells; (3) if, at the ODE step, an imminent bubble collapse is detected, mark the corresponding bubble 
center as 'paused'; no further ODE steps with this bubble will be made until the pause mark is removed; (4) calculate the Cafisch acoustic source terms from the discretized temporal derivative of the bubble volume fractions and apply them to the acoustic pressure (i.e., mass continuity) equation in the corresponding computational cells; and (5) check for cells where the next acoustic rarefaction halfcycle has begun and remove their pause marks.

The acoustic PDEs are solved via the finite-difference time-domain method using specialized 4thorder accurate spatial differencing schemes optimized for acoustic waves, and similarly optimized

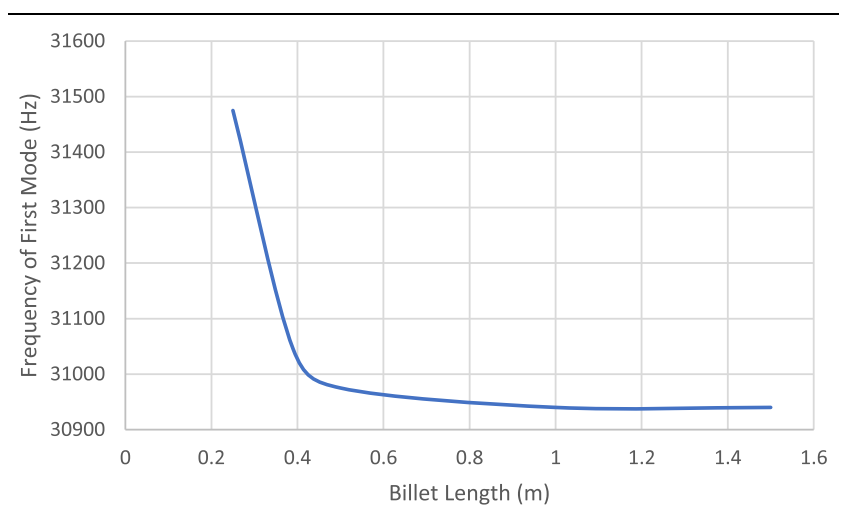

Fig. 8. Effect of billet length on the frequency of the first mode. 3rd-order time-stepping ones. ${ }^{32}$ The time steps needed for stable and accurate acoustic computation with this method are sufficiently small so that the ODE steps can be implemented as simple Euler 1storder forward steps; if needed, more sophisticated ODE methods can be added into the software implementation.

Figure 4, taken from a simulation of sonification of water in an acrylic tank as used in, ${ }^{17}$ illustrates the model behavior by superimposing the calculated local acoustic pressure and bubble radius. The bubble grows many times its original size, $R_{0}$, during intervals of persistent negative pressure, but is not allowed to shrink below $R_{0}$ by the pausing algorithm, thus avoiding the exceptionally small time steps that would be needed if the bubble collapse and rebounds were to be resolved in detail. The bubbles are assumed to have a mean radius of $5 \mu \mathrm{m}$ and a mean concentration of $1.25 \times 10^{8} \mathrm{~m}^{-3}$.

Numerical studies of resonance conditions for contactless liquid metal sonication typically need $0.1 \mathrm{~s}$ simulated intervals in order to achieve $10 \mathrm{~Hz}$ FFT post-processing resolution which would allow accurate prediction of the required driving frequency. The efficiency of the new method presented here allows 3-dimensional simulation runs covering the above requirement to take between 4 and $60 \mathrm{~min}$ on 16-core desktop personal computer

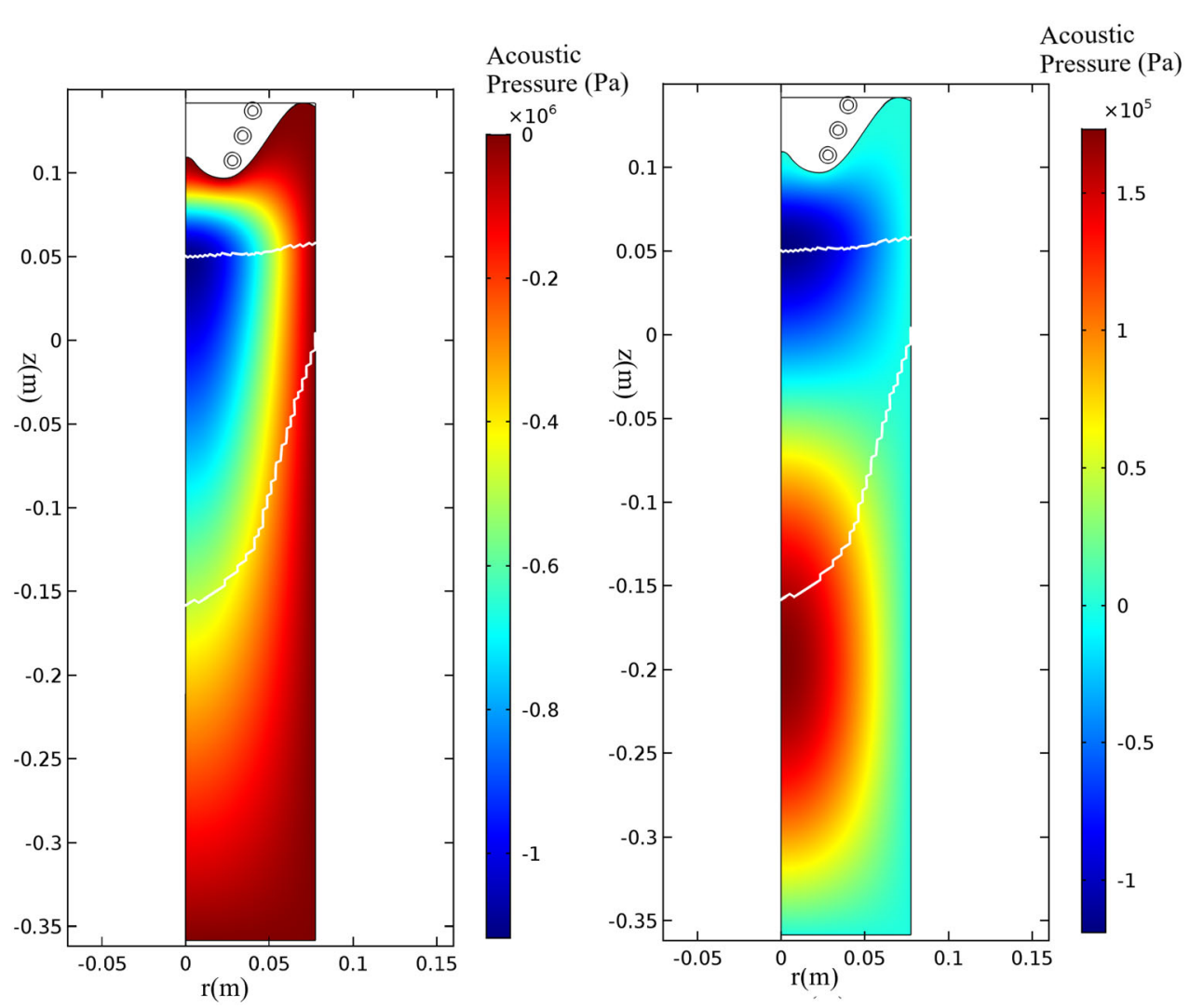

Fig. 9. Plots of the first two modes $(30,940$ and $32,675 \mathrm{~Hz})$ for a billet length of $0.5 \mathrm{~m}$. 


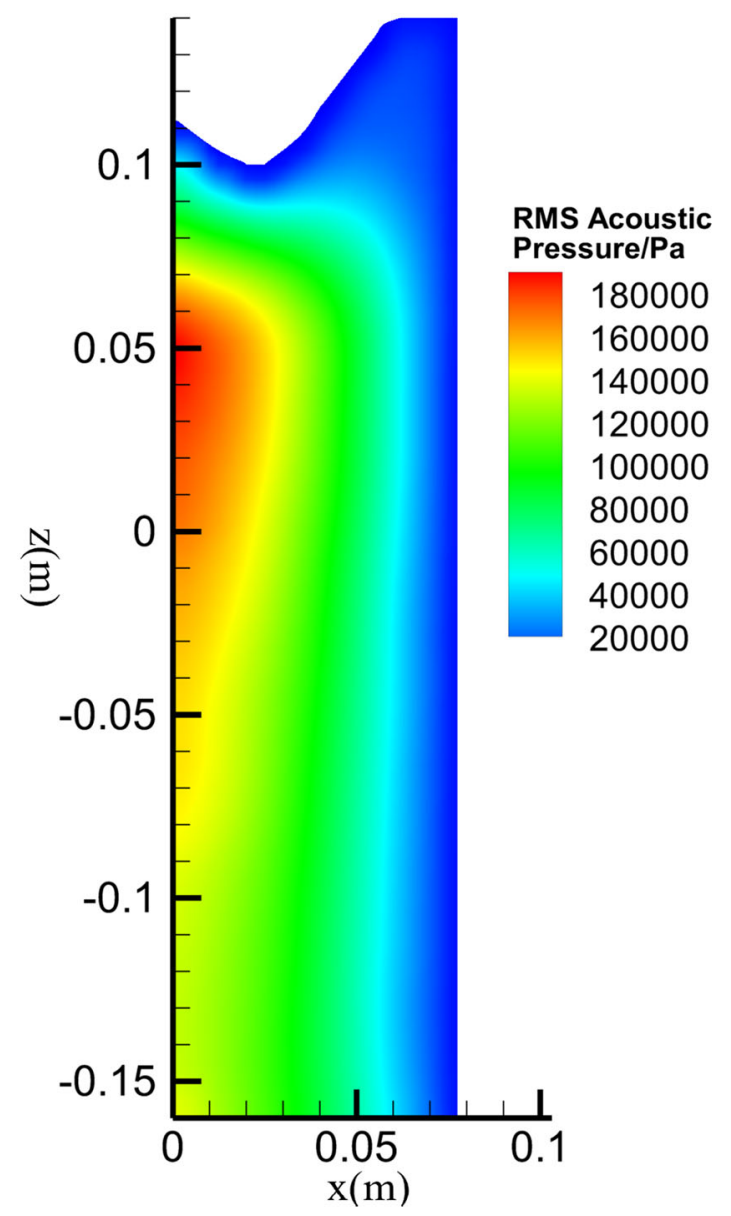

Fig. 10. RMS pressure after $0.055 \mathrm{~s}$ in the transient cavitation model.

depending on mesh density and geometrical features of the cases.

\section{RESULTS AND DISCUSSION}

With the induction coil lowered towards the melt, the surface is depressed due to the Lorentz force, returning to a characteristic cone on the axis where the force drops to zero. ${ }^{24}$ The temperature and flow plot obtained from SPHINX can be seen in Fig. 5. Three fluid circulations form in the melt, two at the top and one at the bottom. The highest magnetic field is located directly below the coil, causing a high Lorentz force pointing downward. This drives the flow deep into the melt, which then circulates back up the edge and center of the billet. The third circulation is primarily in the mushy zone and is much slower, driven by sheer forces from the top circulation and resisted by the microstructure. Due to the thermal transport from stirring, the temperature contours flatten close to the liquidus temperature. Consequently, the solidification front also becomes flattened in this region. However, the bottom circulation also pulls the temperature downwards in the mushy zone, resulting in a large region with a

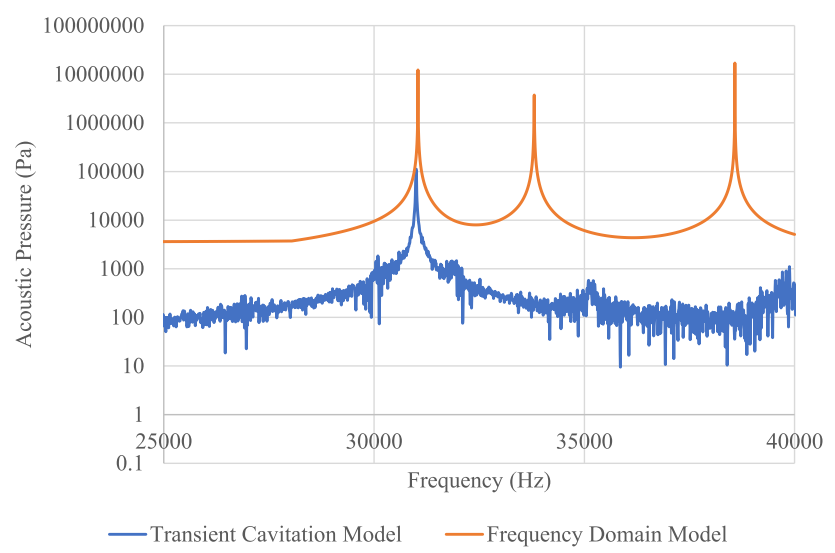

Fig. 11. Comparison the FFT from the transient cavitation model with the frequency response from the frequency domain model. The dominant first mode predicted at $31 \mathrm{kHz}(15.5 \mathrm{kHz}$ electrical) by both methods.

low but non-zero liquid fraction. The non-linear dependence of the liquid fraction on temperature creates a complex spatial variation on the speed of sound, highlighted in Fig. 6.

The frequency responses for various lengths of billet are given in Fig. 7 and show that, once the billet reaches a sufficient length, the first resonant mode tends towards a constant value; this is emphasized in Fig. 8. This suggests that this technique could be used in DC casting to undertake UST in the sump. Figure 9 shows the first two resonant modes for a $0.5-\mathrm{m}$-long billet. The high-pressure region for these modes is concentrated around the liquidus temperature (where, coincidentally, hydrogen is released from solution) and so cavitation is most likely to occur between newly formed dendrites. This would be beneficial from the process point of view, as the shattering of dendrites is identified as one of the mechanisms for grain refinement due to localized cavitation. ${ }^{33}$ Electromagnetic stirring would then distribute any shattered dendrite fragments into the melt, resulting in grain refinement. The $31 \mathrm{kHz}$ resonant frequency predicted with this model is high compared to typical frequencies currently used in UST $(\sim 20 \mathrm{kHz})$; however, by modifying the DC caster geometry and inlet temperatures, the frequency of stable resonant modes can be adjusted.

The cavitation model shows that pressures at the Blake threshold are possible with this technique. Acoustic pressures of $200 \mathrm{kPa}$ are obtained within the melt, and these are then capped due to the energy lost in the cavitation. The model predicts an intermittent pulsed resonance, similar to the behavior experimentally observed in the authors' previous work. ${ }^{20}$ Figure 10 shows the dominant resonant mode obtained at $31 \mathrm{kHz}$, which compares favorably with the resonant model results in Fig. 9. Figure 11 depicts a comparison of the frequency domain response and a fast Fourier transform of the 
time-dependent results. As shown, there is a good match between the models for the first resonant mode. The resonant modes with natural frequencies different from the chosen driving frequency of $31 \mathrm{kHz}$, and predicted by the linear Helmholtz model, appear to be suppressed in the time-dependent case. This is an expected result, as eigenmodes only become active when the driving frequency approaches a corresponding eigenfrequency. Additionally, the pulsating hydrogen bubbles represented in the time-dependent cavitation model cause attenuation of the ultrasound by relieving the overall tension in the liquid during the rarefaction stage of the cycle, further weakening the non-resonant modes.

\section{CONCLUSION}

This initial modeling work has highlighted the potential of using a contactless ultrasonic device for continuous UST in DC casting. A stable resonant mode is achievable in the melt as the billet gets longer. This is thought to be due to the change in the speed of sound as the aluminum solidifies. This will reflect a percentage of the sound back into the melt, achieving resonance. This stable resonant mode in the melt allows for a single excitation frequency to be used as the billet length increases. The location of the high-pressure region of the mode is concentrated around the liquidus temperature, where localized cavitation to this region may significantly enhance the breaking up of dendrites. Combined with transport of the dendrite fragments through electromagnetic stirring, this may lead to favorable conditions for a refined microstructure. As an added benefit, the vigorous stirring equalizes the temperature in the pool and provides for good mixing of the alloy elements, preventing macrosegregation.

This is an ongoing project, and work is in progress to prove the efficacy of this method experimentally and to validate the numerical findings presented here; in particular, the necessary assumption made that the speed of sound varies linearly with the solid fraction. Further modeling work on the acoustics of the system will study resonance modes and their dependencies on key processing parameters. On the flow side, further planned refinements include the use of a viscosity change at the coherency limit to represent a dendritic slurry instead of a Darcy resistance, coupled with a transport model for freeflowing equiaxed dendrites.

\section{ACKNOWLEDGMENTS}

The authors acknowledge financial support from the UK Engineering and Physical Sciences Research Council (EPSRC) through Grants EP/ P034411/1, EP/R000239/1 and EP/R002037/1.

\section{CONFLICT OF INTEREST}

On behalf of all authors, the corresponding author states that there is no conflict of interest.

\section{OPEN ACCESS}

This article is licensed under a Creative Commons Attribution 4.0 International License, which permits use, sharing, adaptation, distribution and reproduction in any medium or format, as long as you give appropriate credit to the original author(s) and the source, provide a link to the Creative Commons licence, and indicate if changes were made. The images or other third party material in this article are included in the article's Creative Commons licence, unless indicated otherwise in a credit line to the material. If material is not included in the article's Creative Commons licence and your intended use is not permitted by statutory regulation or exceeds the permitted use, you will need to obtain permission directly from the copyright holder. To view a copy of this licence, visit http://creative commons.org/licenses/by/4.0/.

\section{REFERENCES}

1. G.I. Eskin and D.G. Eskin, Ultrasonic Treatment of Light Alloy Melts (Boca Raton: Taylor \& Francis, 2017).

2. T. Meek, X. Jian, H. Xu, and Q. Han, Ultrasonic Processing of Materials (Oak Ridge: Oak Ridge National Laboratory, 2006).

3. I. Tzanakis, W.W. Xu, G.S.B. Lebon, D.G. Eskin, K. Pericleous, and P.D. Lee, Phys. Procedia 70, 841 (2015).

4. M. Rappaz, J.M. Drezet, and M. Gremaud, Met. Trans. A 30, 449 (1999).

5. G. Dobra, P. Moldovan, C. Stănică, G. Popescu, and M. Buţu, in TMS Light Met. 2007 (Springer, 2017), pp. 733-737.

6. M. Lalpoor, D.G. Eskin, D. Ruvalcaba, H.G. Fjær, A. Ten Cate, N. Ontijt, and L. Katgerman, Mater. Sci. Eng. A 528, 2831 (2011).

7. K.A. Jackson, J.D. Hunt, D.R. Uhlmann, and T.P. Seward, Trans. Metall. Soc. Aime 236, 149 (1966).

8. H. Nguyen-Thi, G. Reinhart, N. Mangelinck-Noël, H. Jung, B. Billia, T. Schenk, J. Gastaldi, J. Härtwig, and J. Baruchel, Met. Trans. A. 38 A, 1458 (2007).

9. A.L. Greer, P.S. Cooper, M.W. Meredith, W. Schneider, P. Schumacher, J.A. Spittle, and A. Tronche, Adv. Eng. Mater. 5,81 (2003).

10. Z. Fan, Y. Wang, M. Xia, and S. Arumuganathar, Acta Mater. 57, 4891 (2009).

11. X. Liao, Q. Zhai, J. Luo, W. Chen, and Y. Gong, Acta Mater. 55, 3103 (2007).

12. Y.Y. Gong, J. Luo, J.X. Jing, Z.Q. Xia, and Q.J. Zhai, Mater. Sci. Eng. A 497, 147 (2008).

13. J.W. Fu and Y.S. Yang, Mater. Lett. 67, 252 (2012).

14. R. Guan, C. Ji, and M. Zhu, Met. Trans. B 51, 1137 (2020).

15. G. Salloum-Abou-Jaoude, D. G. Eskin, C. Barbatti, P. Jarry, M. Jarrett, and Z. Fan, in TMS Light Met. 2017 (Springer, 2017), pp. 997-1003.

16. G.I. Eskin, Ultrason. Sonochem. 8, 319 (2001).

17. T. Subroto, D. G. Eskin, C. Beckwith, I. Tzanakis, G. Djambazov, and K. Pericleous, in TMS Light Met. 2020 (Springer, 2020), pp. 981-987.

18. G.S.B. Lebon, G. Salloum-Abou-Jaoude, D. Eskin, I. Tzanakis, K. Pericleous, and P. Jarry, Ultrason. Sonochem. 54, 171 (2019).

19. C. E. H. Tonry, G. Djambazov, A. Dybalska, W. D. Griffiths, C. Beckwith, V. Bojarevics, and K. A. Pericleous, Ultrason. Sonochem. 63, (2020).

20. K. Pericleous, V. Bojarevics, G. Djambazov, A. Dybalska, W. D. Griffiths, and C. Tonry, Materials. 12, (2019).

21. V. Bojarevics, G.S. Djambazov, and K.A. Pericleous, Met. Trans. A 46, 2884 (2015).

22. I. Kaldre and A. Bojarevics, JOM 72, 2892 (2020). 
23. V. Hatić, B. Mavrič, N. Košnik, and B. Šarler, Appl. Math. Model. 54, 170 (2018).

24. K. Pericleous and V. Bojarevics, Prog. Comput. Fluid Dyn. 7, 118 (2007)

25. C. Canuto, M.Y. Hussaini, A. Quarteroni, and T.A. Zang, Spectral Methods (Berlin: Springer, 2006).

26. V. Bojarevics, R.A. Harding, K. Pericleous, and M. Wickins, Met. Trans. B 35, 785 (2004).

27. V. Bojarevics, K. Pericleous, and M. Cross, Met. Trans. B 31, 179 (2000).

28. D. Wilcox, Turbulence Modeling for CFD (DCW Industries: La Cãnada, CA, 1998).

29. D. R. Tobergte and S. Curtis, in Ultrasound Technologies for Food Bioprocessing edited by H. Feng, G. V Barbosa-Canovas, and J. Weiss (Berlin: Springer, 2013), pp. 1689-1699.
30. R.E. Caflisch, M.J. Miksis, G.C. Papanicolaou, and L. Ting, J. Fluid Mech. 153, 259 (1985)

31. J.B. Keller and M. Miksis, J. Acoust. Soc. Am. 68, 628 (1980).

32. G.S.B. Lebon, I. Tzanakis, G. Djambazov, K. Pericleous, and D.G. Eskin, Ultrason. Sonochem. 37, 660 (2017).

33. B. Wang, D. Tan, T.L. Lee, J.C. Khong, F. Wang, D. Eskin, T. Connolley, K. Fezzaa, and J. Mi, Data Br. 17, 837 (2018).

Publisher's Note Springer Nature remains neutral with regard to jurisdictional claims in published maps and institutional affiliations. 